\title{
Hans Schemann / Maria Luiza Schemann-Dias / Luisa Amorim-Braun / Teresa Hundertmark-Santos Martins / Maria João Romero Dias Duque-Gitt / Helena Costa, Idiomatik Deutsch-Portugiesisch, unter Mitarbeit von Anette Dirauf,
} Maria Augusta Pimenta, Filomena de Sousa-Möckel, 2., durchgesehene Auflage, Hamburg, Buske, 2012, XXXIX + 1228 p.

\section{Hans Schemann / Idalete Dias, Idiomatik Portugiesisch-Deutsch,} 2., durchgesehene Auflage, Hamburg, Buske, 2013, XIX + 873 p.

Ohne Übertreibung ist die neuerliche Veröffentlichung der deutschen und portugiesischen Idiomatik von Hans Schemann nunmehr unter der Rubrik Lebenswerk $\mathrm{zu}$ sehen, hat sich die Idiomatik doch unter seiner Herausgeberschaft $\mathrm{zu}$ einem polyglotten Großprojekt ausgewachsen. In Kombination mit Deutsch gibt es inzwischen fünf Groß-Idiomatiken: Englisch, Französisch, Italienisch, Portugiesisch und Spanisch. Jetzt gedenkt Schemann - mit Deutsch als Scharniersprache -, daraus ein gewaltiges polyglottes Idiomatikwörterbuch in elektronischer Form vorzubereiten, in welchem man dann quer durch alle Sprachen nach Einträgen suchen kann, denn für alle fünf zweisprachigen Wörterbücher fanden Schemanns Deutsche Idiomatik (Pons, 1993) sowie das Synonymwörterbuch deutscher Redensarten (Pons, 1992) als Ausgangsmaterial Verwendung. In Zeiten, da neue Wörterbücher auf dem Markt als Druckprodukt eine Rarität geworden sind, kann man dem Buske-Verlag nicht genug Respekt zollen für dieses Vorhaben.

Die Idiomatik Deutsch-Portugiesisch von Hans Schemann stellt die fast unveränderte Fassung des gleichen Titels bei Pons 2002 dar. Laut Angaben des Autors wurden einige Fehler behoben und Währungsangaben aktualisiert, so dass das Werk ohne veränderten Umbruch herausgebracht werden konnte. Das hat zur Folge, dass das Wörterbuch in der alten Rechtschreibung erschien, mit den entsprechenden orthografischen Folgen: ß/ss-Problematik, die Mehrfachkonsonanten: stillegen, numerieren. Gleiches gilt für die portugiesische Orthografie.

Eine ausführliche Einleitung in Deutsch und Portugiesisch, in welcher die Materialbasis, der Begriff Idiomatik, Artikelaufbau und Verweisstruktur erklärt werden, ist dem eigentlichen Korpus vorangestellt. Dieses enthält 33.000 Wendungen, gesammelt aus literarischen Werken sowie Presseerzeugnissen des 20. Jh. und idiomatischen Nachschlagewerken.

Bei dieser Datenfülle verwundert es nicht, dass das über 1.200 Seiten umfassende eigentliche Wörterbuch für den Nutzer eine Herausforderung darstellt, denn besonders die umfangreicheren Stichwortartikel (Stichwort Kopf geht über achteinhalb Seiten und Hand/Hände gar über elfeinhalb Seiten) sind trotz unterschiedlicher, aber insgesamt recht kleiner Schriftgrößen zur Unterscheidung von 
Stichwort, Äquivalent und Beispielsätzen (in deutscher Sprache) kaum übersichtlich, und das Gesuchte ist nur schwer aufzufinden. Bei einer solchen Datenmenge ist es allerdings schwer zu raten, wie man die einzelnen Artikel optisch besser gestalten könnte. Hier würde ganz sicher eine elektronische Version sehr hilfreich sein; man vergleiche einmal das größte einsprachige Wörterbuch der portugiesischen Sprache - Dicionário Houaiss da língua portuguesa - in der gedruckten und der elektronischen Version mit seiner Mehrfarbigkeit und der großzügigeren grafischen Darstellung. Zusätzlich erschwert wird die Transparenz eines Artikels häufig durch per Schrägstrich oder Klammer getrennte Lemmavarianten oder Teile davon. Natürlich ist es grundsätzlich gut, dass Varianten angegeben werden; das macht aber das rasche Identifizieren des gesuchten Idiomatismus nicht eben leichter.

Beispiele:

(jm.) etw. in den schwärzesten/ dunkelsten/ (schwarzen)/ (dunklen) Farben ausmalen/ (schildern)

gezeigt: dem/der/dem Herrn Schulze/ ... habe ich(hat er/...) es aber gezeigt!

Als Auswahlkriterium wählte der Autor einen «bewußt weit» gefassten Idiomatikbegriff. Das hat zur Folge, dass der Nutzer auf recht viele Einträge stößt, die vordergründig kaum etwas mit Idiomatik zu tun haben, wenn man unter Idiomatik versteht, dass die gewählte sprachliche Einheit eine gewisse Idiomatizität aufweist, d.h. dass die Wortgruppe zumindest ansatzweise opak, undurchsichtig ist und das Gesagte nicht das Gemeinte ist. Beispiel: mit der Tür ins Haus fallen.

Dem kann entgegengehalten werden, dass es immer von Vorteil ist, als Lexikograf auch an den Nichtmuttersprachler zu denken, der u.U. nicht wissen kann, wie bestimmte Mehrwortverbindungen üblicherweise gebildet werden.

Beispiele:

die Hände aus der Tasche nehmen, die Hände in die Tasche stecken, kalte Küche, im Rohbau sein, in meiner Abwesenheit.

Aber Idiomatismen sind dies ganz sicher nicht. Wenn man aber das Konzept so ausweitet, dann muss freilich angemerkt werden, dass sehr viele solcher festen, nicht idiomatischen Mehrwortverbindungen eben nicht enthalten sind, und auch nicht enthalten sein können. Das Ergebnis hätte nicht zwischen zwei Buchdeckel gepasst. Und eine solch umfangreiche Bearbeitung dieser Thematik dürfte im Rahmen eines idiomatischen Wörterbuches auch nicht leistbar sein. Aus unserer Sicht hätte diese Kategorie von Einträgen zu Gunsten der eigentlichen Idiomatismen «außen vor» bleiben sollen; immer im Interesse der Nutzerfreundlichkeit.

Dadurch, dass in die deutsche Wortliste auch Schemanns Synonymwörterbuch deutscher Redensarten eingearbeitet wurde, trifft der Nutzer sehr häufig auf Querverweise zu anderen, synonymen oder quasi synonymen Stichwörtern. 


\author{
Beispiele: \\ (einen) klaren Kopf bewahren - ruhig'/(kaltes) Blut bewahren; \\ der/die Erna/... hat (ja/...) ein Gesicht - da braucht einer/man 'nen Waffenschein $v u l g$ - \\ ein Gesicht zum Reinhauen/Reinschlagen haben; \\ Ast: sich auf dem absteigenden Ast befinden/(auf ... sein) - es geht bergab mit jm./etw.
}

Wir meinen, dass es kaum mehr Platz benötigt hätte, jeweils das portugiesische Äquivalent anstatt des deutschen Querverweises einzufügen, das ganze Wörterbuch wäre sehr viel nutzerfreundlicher ausgefallen. Nur um ein Beispiel zu geben: Sucht man nach der Wendung seine Hand im Spiel haben, muss man erst bis zur achten Seite des Schlagwortes Hand gelangen, um dann auf Finger verwiesen zu werden.

Allemal Ansichtssache dürfte es wohl sein, wenn es um Einträge geht, die bereits vom Autor als veraltend oder selten markiert wurden. Hier fragt man sich, ob nicht ein Weniger an solchen Einträgen die Möglichkeit erbracht hätte, das Korpus zu entschlacken. Natürlich kann man dagegen argumentieren, dass, wer einen älteren Text liest und dabei auf solche Idiomatismen stößt, dankbar sein dürfte, diese im Idiomatikwörterbuch nachschlagen zu können.

\title{
Beispiele:
}

Stint: sich freuen wie ein Stint ugs veraltend - sich freuen wie ein Schneekönig;

in den Stock legen - hist selten amarrar alg.... Hier wird dem deutschen Nutzer u.U. erst durch das portugiesische Äquivalent der Inhalt der Wendung klar; ähnlich im Stock sitzen; an den Hungerpfoten saugen sal selten - Hunger leiden.

Sicher ist es immer auch etwas subjektiv zu entscheiden, was als selten zu bezeichnen ist.

Beispiel:

jm. ein Ohr abkauen oder lange Ohren machen scheinen uns durchaus gebräuchliche Wendungen zu sein.

Unter Umständen mag es im Deutschen auch jüngere, synonyme Wendungen zu den mit selten markierten Wendungen im Wörterbuch geben:

Jacke: das/etw. ist eine alte Jacke ugs selten ser mais (do) que sabido.

Hier böte sich - angeregt durch das portugiesische Äquivalent - die deutsche Wendung sattsam bekannt sein an.

Dieses Korpus entstand über viele Jahre hinweg (Schemanns erstes idiomatisches Wörterbuch datiert von 1979). Die hier besprochene Großidiomatik ist und bleibt ein gewaltiger Fundus an sprachlichem Material, das dem Nutzer zahllose Wendungen in der Zielsprache erschließt. Betrachtet man die Situation am Wör- 
terbuchmarkt, dürfte es nicht ganz abwegig sein anzunehmen, dass dieses Werk ein Solitär bleiben wird.

Die beiden hier besprochenen Wörterbücher wurden gemeinsam rezensiert, da sie der Logik eines Ausgangsbandes für die Sprachrichtung Deutsch-Portugiesisch und dem entsprechenden Umkehrband Portugiesisch-Deutsch folgen. Diese Struktur erschließt sich dem Nutzer nicht sofort, da man von einem Wörterbuch mit der Überschrift Idiomatik Portugiesisch-Deutsch prinzipiell erwartet, dass dieses von den Idiomatismen der portugiesischen Sprache ausgeht. Der Autor verweist zwar in seiner Einführung auf das mit seiner Frau herausgegebene Wörterbuch Portugiesisch-deutsche Idiomatik, das sehr wohl von dem Portugiesischen ausgeht, aber der Unterschied zwischen diesem Wörterbuch und dem hier rezensierten Umkehrband springen dem Nutzer nicht unbedingt ins Auge.

Im Umkehrband selbst ist diese Eigenschaft im Vorwort und in der Einführung erläutert; aus dem Buchtitel wird diese Konzeption jedoch nicht ersichtlich. Es wäre aus Nutzersicht jedoch wichtig, dass genau diese Eigenschaft noch deutlicher herausgestellt würde, weil sich daraus erstens die Erklärung für den deutlich geringeren Umfang des Umkehrbandes ergibt (der Autor verweist für die Erläuterung der deutschen Idiomatismen auf den Ausgangsband) und damit zweitens der Frustration über schwer auffindbare oder nicht gefundene Einträge zu portugiesischen Idiomatismen entgegengewirkt werden kann. So stellt sich dem Nutzer die Nachschlagearbeit im Umkehrband etwas mühevoller dar, weil er nicht der Logik eines herkömmlichen Wörterbuches folgt.

Auch wenn Nichtmuttersprachler die deutschen Abkürzungen fam [Familiensprache], lit [literarisch] oder iron [ironisch] vermutlich verstehen, fehlen im Umkehrband die entsprechenden Erläuterungen dazu. Die Abkürzungen ugs [umgangssprachlich], path [pathetisch] und sal [salopp] sind jedoch für Nichtmuttersprachler nicht ohne weiteres erschließbar. Ob die Abkürzung path [pathetisch] auch jedem deutschsprachigen Nutzer auf Anhieb einleuchtet, sei dahingestellt:

acordar $^{2}$ - aus dem $\underline{\text { Schlaf reißen path }}$

Fazit: Die aufgezeigten Lücken und Grenzen dieser Werke schmälern in keiner Weise die große Leistung des Autors, in jahrelanger und mühseliger lexikografischer Arbeit deutsche Idiome zusammengestellt zu haben und sich weiterhin für das auf dem Wörterbuchmarkt so vernachlässigte Sprachenpaar Deutsch/Portugiesisch zu engagieren. Die Tatsache, dass sich überhaupt noch ein Verlag so stark für die deutsch-portugiesische Lexikografie einsetzt, muss ebenfalls lobend hervorgehoben werden. Trotz aller Unzulänglichkeiten sind beide Bände für fortgeschrittene Sprachlerner, Übersetzer und alle anderen, die sich beruflich oder 
privat mit diesem Sprachenpaar beschäftigen, eine wertvolle Handreichung. Eine ergänzende elektronische Fassung ist aus Sicht der Nutzer sehr wünschenswert.

Dipl. Lateinam. Lutz Hoepner: Humboldt-Universität zu Berlin, Institut für Romanistik, Unter den Linden 6,10099 Berlin, E-Mail: Ihoepner@t-online.de

Prof. Dr. Tinka Reichmann: Universidade de São Paulo, Departamento de Letras Modernas, Av. Prof. Luciano Gualberto, 403, BR-05508-900 São Paulo, E-Mail: reichmann@usp.br 